\title{
INDUCTION FUNCTORS FOR GROUP CORINGS
}

\author{
Quan-Guo Chen and Ding-Guo Wang*
}

\begin{abstract}
In the paper, we prove that the induction functor stemming from every morphism of group coring versus coring has a left adjoint, called ad-induction functor. The separability of the induction functor is characterized, extending some results for corings.
\end{abstract}

\section{Introduction}

As the generalization of coring, introduced by Sweedler [8] and revised by Brzeziński [1], Caenepeel et al. introduced the group coring and developed Galois theory for group corings in [2], which have become increasingly an interesting subject to study. Some study of the new structure has been carried out in recent papers (see [4], [6] and [10]).

Given an $A$-coring $C$, where $A$ is an algebra over a fixed field $k$, we have the category $\mathscr{M}^{C}$ of all the right comodule over $C$. It follows from [1] that there exists a pair of adjoint functor between the category $\mathscr{M}^{C}$ and the category $\mathscr{M}_{A}$ of all the right $A$-modules. The extension of this result to the context of Hopf group-coalgebras was made in the work of the authors in [10], that is, there exists a pair of adjoint functor between the category $\mathscr{M}^{G, C}$ of all the right $G$ - $C$-comodule and the category $\mathscr{M}_{A}$ of all the right $A$-modules. As we know, an algebra $A$ has a canonical $A$-coring structure over itself. A natural question occurs to us: whether there exists a pair of adjoint functor between the category $\mathscr{M}^{G, C}$ of all the right $G$-C-comodule and the category $\mathscr{M}^{D}$ of all the right comodules over a given $B$-coring, if so, how to characterize its separability. This is done in this paper.

This paper is organized as follows.

2000 Mathematics Subject Classification. 16W30.

Key words and phrases. group coring, comodule, separable functor.

* Corresponding author.

The work was supported by the National Natural Science Foundation of China (No. 11261063 and 11471186), the Foundation for Excelent Youth Science and Technology Innovation Talents of Xin Jiang Uygur Autonomous Region (No. 2013721043).

Received March 5, 2014; revised June 20, 2014. 
In Section 2, we recall some basic concepts such as group coring and cotensor product. In Section 3, we use the notion of homomorphism of corings to construct a pair of adjoint functors (the induction functor and its adjoint, called here ad-induction functor). Finally, the separability of the induction functor is characterized.

\section{Preliminaries} field.

Throughout this paper, we always let $G$ be a group with the unit $e$ and $k$ a

2.1. Group corings. First recall from [2] that a $G$-group $A$-coring (or shortly a $G$-A-coring) $C$ is a family $\left\{C_{\alpha}\right\}_{\alpha \in G}$ of $A$-bimodules together with a family of $A$-bimodule maps

$$
\Delta_{\alpha, \beta}: C_{\alpha \beta} \rightarrow C_{\alpha} \otimes_{A} C_{\beta}, \quad \varepsilon: C_{e} \rightarrow A
$$

such that

$$
\left(\Delta_{\alpha, \beta} \otimes_{A} C_{\gamma}\right) \circ \Delta_{\alpha \beta, \gamma}=\left(C_{\alpha} \otimes_{A} \Delta_{\beta, \gamma}\right) \circ \Delta_{\alpha, \beta \gamma}
$$

and

$$
\left(C_{\alpha} \otimes_{A} \varepsilon\right) \circ \Delta_{\alpha, e}=C_{\alpha}=\left(\varepsilon \otimes_{A} C_{\alpha}\right) \circ \Delta_{e, \alpha}
$$

for all $\alpha, \beta, \gamma \in G$.

Remark 2.1. If $C$ is a $G$-A-coring, then $C_{e}$ is an ordinary $A$-coring in sense of $[8]$.

We use the following Sweedler-type notation for the comultiplication maps $\Delta_{\alpha, \beta}$ :

$$
\Delta_{\alpha, \beta}(c)=c_{(1, \alpha)} \otimes_{A} c_{(2, \beta)}
$$

for all $c \in C_{\alpha \beta}$.

A right $G$-C $C$-comodule $M=\left\{M_{\alpha}\right\}_{\alpha \in G}$ is a family of right $A$-modules, together with a family of right $A$-linear maps $\rho^{M}=\left\{\rho_{\alpha, \beta}^{M}\right\}_{\alpha, \beta \in G}$,

$$
\rho_{\alpha, \beta}^{M}: M_{\alpha \beta} \rightarrow M_{\alpha} \otimes_{A} C_{\beta}
$$

such that

$$
\left(M_{\alpha} \otimes_{A} \Delta_{\beta, \gamma}\right) \circ \rho_{\alpha, \beta \gamma}^{M}=\left(\rho_{\alpha, \beta}^{M} \otimes_{A} C_{\gamma}\right) \circ \rho_{\alpha \beta, \gamma}^{M}
$$

and

$$
\left(M_{\alpha} \otimes_{A} \varepsilon\right) \circ \rho_{\alpha, e}^{M}=M_{\alpha}
$$

for all $\alpha, \beta, \gamma \in G$. 
We use the following Sweedler-type notation:

$$
\rho_{\alpha, \beta}^{M}(m)=m_{[0, \alpha]} \otimes_{A} m_{[1, \beta]}
$$

for $m \in M_{\alpha \beta}$.

A morphism between two right $G$-C-comodules $M$ and $N$ is a family of right $A$-linear maps $f=\left\{f_{\alpha}: M_{\alpha} \rightarrow N_{\alpha}\right\}_{\alpha \in G}$ such that

$$
\left(f_{\alpha} \otimes_{A} C_{\beta}\right) \circ \rho_{\alpha, \beta}=\rho_{\alpha, \beta} \circ f_{\alpha, \beta} .
$$

The category of right $G$-C-comodules will be denoted by $\mathscr{M}^{G, C}$.

2.2. The cotensor product. Let $D$ be a $B$-coring. Let $M \in \mathscr{M}^{D}$ and $N \in^{D} \mathscr{M}$. First recall that the cotensor product $M \square_{D} N$ of $M$ and $N$ is given by

$$
\begin{gathered}
M \square_{D} N=\left\{\sum_{i} m_{i} \otimes_{B} n_{i} \in M \otimes N \mid \sum_{i} m_{i[0]} \otimes_{B} m_{i[1]} \otimes_{B} n_{i}\right. \\
\left.=\sum_{i} m_{i} \otimes_{B} n_{i[-1]} \otimes_{B} n_{i[0]}\right\},
\end{gathered}
$$

that is, $M \square_{D} N$ fits an exact sequence

$$
0 \rightarrow M \square_{D} N \rightarrow M \otimes_{B} N \rightrightarrows M \otimes_{B} D \otimes_{B} N
$$

where the two maps $M \otimes_{B} N \rightarrow M \otimes_{B} D \otimes_{B} N$ are $\rho^{M} \otimes_{B} N$ and $M \otimes_{B} \rho^{N}$.

\section{Separable homomorphisms of $G$ - $A$-corings versus corings}

Consider a $G$-A-coring $C$ and a $B$-coring $D$, where $A$ and $B$ are both $k$-algebra.

Definition 3.1. A coring homomorphism is a pair $(\varphi, \mu)$, where $\mu: A \rightarrow B$ is a homomorphism of algebras and $\varphi: C_{e} \rightarrow D$ is a homomorphism of $A$-bimodules, and such that the following equations

$$
\begin{gathered}
\vartheta_{D, D} \circ\left(\varphi \otimes_{A} \varphi\right) \circ \Delta_{e, e}=\Delta_{D} \circ \varphi, \\
\mu \circ \varepsilon_{e}=\varepsilon_{D} \circ \varphi,
\end{gathered}
$$

where $\vartheta_{D, D}: D \otimes_{A} D \rightarrow D \otimes_{B} D$ is the canonical map induced by $\mu$.

Throughout the rest of this section, we always assume that there exists a coring homomorphism $(\varphi, \mu)$ between two corings $C_{e}$ and $D$.

\subsection{The induction functor.}

Proposition 3.2. The assignment $M \mapsto M_{e} \otimes_{A} B$ establishes a functor $(-)_{e} \otimes_{A} B: \mathscr{M}^{G, C} \rightarrow \mathscr{M}^{D}$. 
Define

Proof. Let $\rho^{M}=\left\{\rho_{\alpha, \beta}^{M}: M_{\alpha, \beta} \rightarrow M_{\alpha} \otimes_{A} C_{\beta}\right\}_{\alpha, \beta \in G}$ be a right $G$-C-comodule.

$$
\rho^{M_{e} \otimes_{A} B}: M_{e} \otimes_{A} B \rightarrow M_{e} \otimes_{A} B \otimes_{B} D, \quad m \otimes_{A} b \mapsto m_{[0, e]} \otimes_{A} 1_{B} \otimes_{B} \varphi\left(m_{[1, e]}\right) \cdot b .
$$

It is straightforward to check that $M_{e} \otimes_{A} B$ is an object of $\mathscr{M}^{D}$. In order to show the assignment $M \mapsto M_{e} \otimes_{A} B$ is functorial, we will prove that $f_{e} \otimes_{A} B$ is a homomorphism of right $D$-comodules for every morphism $f=\left\{f_{\alpha}: M_{\alpha} \rightarrow N_{\alpha}\right\}_{\alpha \in G}$ in $\mathscr{M}^{G, C}$. In fact, for all $m \in M_{e}$ and $b \in B$, we have

$$
\begin{aligned}
\rho^{N_{e} \otimes_{A} B} \circ\left(f_{e} \otimes_{A} B\right)\left(m \otimes_{A} b\right) & =f(m)_{[0, e]} \otimes_{A} 1_{B} \otimes_{B} \varphi\left(f(m)_{[1, e]}\right) \cdot b \\
& =f\left(m_{[0, e]}\right) \otimes_{A} 1_{B} \otimes_{B} \varphi\left(m_{[1, e]}\right) \cdot b \\
& =\left(f_{e} \otimes_{A} B \otimes_{B} D\right) \circ \rho^{M_{e} \otimes_{A} B}\left(m \otimes_{A} b\right) .
\end{aligned}
$$

This ends the proof.

3.2. The ad-induction functor. For each $\alpha \in G$, define $\rho^{B \otimes_{A} C_{\alpha}}: B \otimes_{A} C_{\alpha} \rightarrow D \otimes_{B} B \otimes_{A} C_{\alpha}, \quad b \otimes_{A} c \mapsto b \cdot \varphi\left(c_{(1, e)}\right) \otimes_{B} 1_{B} \otimes_{A} c_{(2, \alpha)}$.

Lemma 3.3. $B \otimes_{A} C=\left\{B \otimes_{A} C_{\alpha}\right\}_{\alpha \in G}$ is a D-C-bicomodule.

Proof. It is sufficient to prove that the following diagram is commutative,

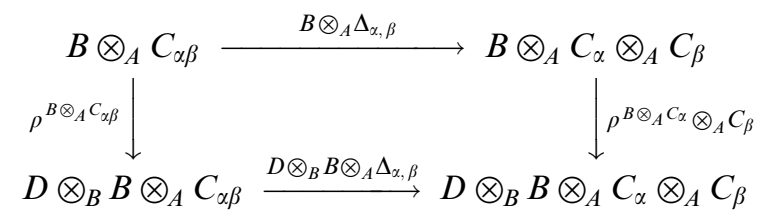

Indeed, for all $b \in B$ and $c \in C_{\alpha \beta}$,

$$
\begin{aligned}
& \left(\rho^{B \otimes_{A} C_{\alpha}} \otimes_{A} C_{\beta}\right) \circ\left(B \otimes_{A} \Delta_{\alpha, \beta}\right)\left(b \otimes_{A} c\right) \\
& =b \cdot \varphi\left(c_{(1, \alpha)(1, e)}\right) \otimes_{B} 1_{B} \otimes_{A} c_{(1, \alpha)(2, \alpha)} \otimes_{A} c_{(2, \beta)} \\
& =b \cdot \varphi\left(c_{(1, e)}\right) \otimes_{B} 1_{B} \otimes_{A} c_{(2, \alpha \beta)(1, \alpha)} \otimes_{A} c_{(2, \alpha \beta)(2, \beta)} \\
& =\left(D \otimes_{B} B \otimes_{A} \Delta_{\alpha, \beta}\right) \circ \rho^{B \otimes_{A} C_{\alpha \beta}}\left(b \otimes_{A} c\right) .
\end{aligned}
$$

This shows that $B \otimes_{A} C=\left\{B \otimes_{A} C_{\alpha}\right\}_{\alpha \in G}$ is a $D$-C-bicomodule.

Proposition 3.4. We have a pair of adjoint functors $(F, U)$ between the categories $\mathscr{M}^{G, C}$ and $\mathscr{M}_{B}$ (the category of right B-module). 
Proof. Take $M=\left\{M_{\alpha}\right\}_{\alpha \in G}$, and define

$$
F: \mathscr{M}^{G, C} \rightarrow \mathscr{M}_{B}, \quad M \mapsto M_{e} \otimes_{A} B .
$$

For a morphism $f=\left\{f_{\alpha}: M_{\alpha} \rightarrow M_{\alpha}^{\prime}\right\}_{\alpha \in G}$ in $\mathscr{M}^{G, C}$, we simply define

$$
F(f)=f_{e} \otimes_{A} B .
$$

Let us now define $U$. For $N \in \mathscr{M}_{B}$, and define

$$
U: \mathscr{M}_{B} \rightarrow \mathscr{M}^{G, C}, \quad N \mapsto N \otimes_{B}\left(B \otimes_{A} C\right),
$$

where $N \otimes_{B}\left(B \otimes_{A} C\right)=\left\{N \otimes_{B}\left(B \otimes_{A} C_{\alpha}\right)\right\}_{\alpha \in G}$ with the $G$-comodule structure maps

$$
\rho^{G(N)}=\left\{\rho_{\alpha, \beta}^{G(N)}=N \otimes_{B}\left(B \otimes_{A} \Delta_{\alpha, \beta}\right)\right\}_{\alpha, \beta \in G} .
$$

Consider the map

$$
\phi: \operatorname{Hom}_{B}\left(M_{e} \otimes_{A} B, N\right) \rightarrow \operatorname{Hom}^{C}\left(M, N \otimes_{B}\left(B \otimes_{A} C\right)\right),
$$

sending $f$ to $\phi(f)=\left\{\phi(f)_{\alpha}\right\}_{\alpha \in G}$, where

$$
\phi(f)_{\alpha}: M_{\alpha} \rightarrow N \otimes_{B}\left(B \otimes_{A} C_{\alpha}\right), \phi(f)_{\alpha}(m)=f\left(m_{[0, e]} \otimes_{A} 1_{B}\right) \otimes_{B}\left(1_{B} \otimes_{A} m_{[1, \alpha]}\right)
$$

and

$$
\varphi: \operatorname{Hom}^{C}\left(M, N \otimes_{B}\left(B \otimes_{A} C\right)\right) \rightarrow \operatorname{Hom}_{B}\left(M_{e} \otimes_{A} B, N\right), g \rightarrow \varphi(g),
$$

where

$$
\varphi(g)\left(m \otimes_{A} b\right)=\left(N \otimes_{B}\left(B \otimes_{A} \varepsilon\right)\left(g_{e}(m)\right)\right) \cdot b .
$$

Let us check that $\phi$ and $\varphi$ are mutually inverse:

$$
\begin{aligned}
& \phi(\varphi(g))_{\alpha}(m)=\varphi(g)\left(m_{[0, e]} \otimes_{A} 1_{B}\right) \otimes_{B}\left(1_{B} \otimes_{A} m_{[1, \alpha]}\right) \\
& =\left(N \otimes_{B}\left(B \otimes_{A} \varepsilon\right)\left(g_{e}\left(m_{[0, e]}\right)\right)\right) \otimes_{B}\left(1_{B} \otimes_{A} m_{[1, \alpha]}\right) \\
& =\left(\left(N \otimes_{B}\left(B \otimes_{A} \varepsilon\right)\right) \otimes_{B}\left(B \otimes_{A} C_{\alpha}\right)\right)\left(g_{e}\left(m_{[0, e]}\right) \otimes_{B} 1_{B} \otimes_{A} m_{[1, \alpha]}\right) \\
& =\left(\left(N \otimes_{B}\left(B \otimes_{A} \varepsilon\right)\right) \otimes_{B}\left(B \otimes_{A} C_{\alpha}\right)\right)\left(g_{\alpha}(m)_{[0, e]} \otimes_{B} 1_{B} \otimes_{A} g_{\alpha}(m)_{[1, \alpha]}\right) \\
& \left(g_{\alpha}(m)=n_{i} \otimes_{B}\left(b_{i} \otimes_{A} c_{i}\right) \in N \otimes_{B}\left(B \otimes_{A} C_{\alpha}\right)\right) \\
& =\left(\left(N \otimes_{B}\left(B \otimes_{A} \varepsilon\right)\right) \otimes_{B}\left(B \otimes_{A} C_{\alpha}\right)\right)\left(n_{i} \otimes_{B}\left(b_{i} \otimes_{A} c_{i(1, e)}\right) \otimes_{B} 1_{B} \otimes_{A} c_{i(2, \alpha)}\right) \\
& =n_{i} \cdot b_{i} \mu\left(\varepsilon\left(c_{i(1, e)}\right)\right) \otimes_{B} 1_{B} \otimes_{A} c_{i(2, \alpha)} \\
& =n_{i} \otimes_{B} b_{i} \otimes_{A} \varepsilon\left(c_{i(1, e)}\right) \cdot c_{i(2, \alpha)} \\
& =n_{i} \otimes_{B} b_{i} \otimes_{A} c_{i}=g_{\alpha}(m)
\end{aligned}
$$

For all $m \in M_{\alpha}$, and 


$$
\begin{aligned}
\varphi \circ \phi(f)\left(m \otimes_{A} b\right) & =\left(N \otimes_{B}\left(B \otimes_{A} \varepsilon\right)\left(\phi(f)_{e}(m)\right)\right) \cdot b \\
& =\left(N \otimes_{B}\left(B \otimes_{A} \varepsilon\right)\left(f\left(m_{[0, e]} \otimes_{A} 1_{B}\right) \otimes_{B}\left(1_{B} \otimes_{A} m_{[1, e]}\right)\right) \cdot b\right. \\
& =f\left(m_{[0, e]} \otimes_{A} 1_{B}\right) \cdot \mu\left(\varepsilon\left(m_{[1, e]}\right)\right) b \\
& =f\left(m_{[0, e]} \cdot \varepsilon\left(m_{[1, e]}\right) \otimes_{A} b\right)=f\left(m \otimes_{A} b\right) .
\end{aligned}
$$

This ends the proof.

For a $G$ - $A$-coring $C$, recall from $[10$, Lemma 3.1] that there exists a pair of adjoint functors $\left(F_{1}, U_{1}\right)$ between the categories $\mathscr{M}^{G, C}$ and $\mathscr{M}_{A}$ (the category of right $A$-modules). Notice that the adjoint functors $(F, U)$ in Proposition 3.4 are the composition of the functors $\left(F_{1}, U_{1}\right)$ and the restriction/induction functor induced by $A \rightarrow B$ :

$$
\mathscr{M}^{G, C} \leftrightarrows \mathscr{M}_{A} \leftrightarrows \mathscr{M}_{B}
$$

Next, take $N \in \mathscr{M}^{D}$ and $N \square_{D}\left(B \otimes_{A} C_{\alpha}\right)$ denotes the cotensor product of $N$ and $B \otimes_{A} C_{\alpha}$. Let $N \square_{D}\left(B \otimes_{A} C\right)=\left\{N \square_{D}\left(B \otimes_{A} C_{\alpha}\right)\right\}_{\alpha \in G}$. From the proof of Prop. 3.4, we have

Proposition 3.5. If $C$ is flat as a left A-module (means that each $C_{\alpha}$ is flat), and $N \in \mathscr{M}^{D}, M=\left\{M_{\alpha}\right\}_{\alpha \in G} \in \mathscr{M}^{G, C}$ then $N \square_{D}\left(B \otimes_{A} C\right)=\left\{N \square_{D}\left(B \otimes_{A} C_{\alpha}\right)\right\}_{\alpha \in G}$ is an object of $\mathscr{M}^{G, C}$ via the structure map $\left\{N \otimes_{B} B \otimes_{A} \Delta_{\alpha, \beta}\right\}_{\alpha, \beta \in G}$. (3.1) restricts to an isomorphism

$$
\operatorname{Hom}^{D}\left(M_{e} \otimes_{A} B, N\right) \cong \operatorname{Hom}^{C}\left(M, N \square_{D}\left(B \otimes_{A} C\right)\right) .
$$

Therefore, $-\square_{D}\left(B \otimes_{A} C\right)$ is right adjoint to $(-)_{e} \otimes_{A} B$.

Proof. We have to show that, for all $\sum_{i} n_{i} \otimes_{B}\left(b_{i} \otimes c_{i}\right) \in N \square_{D}\left(B \otimes_{A} C_{\alpha \beta}\right)$ with $\alpha, \beta \in G$ :

$$
x=\sum_{i}\left(n_{i} \otimes_{B}\left(b_{i} \otimes_{A} c_{i(1, \alpha)}\right)\right) \otimes c_{i(2, \beta)} \in\left(N \square_{D}\left(B \otimes_{A} C_{\alpha}\right)\right) \otimes_{A} C_{\beta} .
$$

For each $\alpha \in G$, we have an exact sequence

$$
0 \rightarrow N \square_{D}\left(B \otimes_{A} C_{\alpha}\right) \rightarrow N \otimes_{B}\left(B \otimes_{A} C_{\alpha}\right) \rightrightarrows N \otimes_{B} D \otimes_{B}\left(B \otimes_{A} C_{\alpha}\right) .
$$

Since $C_{\beta}$ is flat, we have another exact sequence

$$
\begin{aligned}
0 \rightarrow\left(N \square_{D}\left(B \otimes_{A} C_{\alpha}\right)\right) \otimes_{A} C_{\beta} & \rightarrow N \otimes_{B}\left(B \otimes_{A} C_{\alpha}\right) \otimes_{A} C_{\beta} \\
& \rightrightarrows N \otimes_{B} D \otimes_{B}\left(B \otimes_{A} C_{\alpha}\right) \otimes_{A} C_{\beta} .
\end{aligned}
$$

Therefore, in order to show that $x \in\left(N \square_{D}\left(B \otimes_{A} C_{\alpha}\right)\right) \otimes_{A} C_{\beta}$, it suffices to show that

$$
\left(\rho^{N} \otimes_{B} B \otimes_{A} C_{\alpha} \otimes_{A} C_{\beta}\right)(x)=\left(N \otimes_{B} \rho^{B \otimes_{A} C_{\alpha}} \otimes_{A} C_{\beta}\right)(x) .
$$


Indeed, we have

$$
\begin{aligned}
\left(\rho^{N} \otimes_{B} B \otimes_{A} C_{\alpha} \otimes_{A} C_{\beta}\right)(x) & =\sum_{i} n_{i(0)} \otimes_{B} n_{i(1)} \otimes_{B} b_{i} \otimes_{A} c_{i(1, \alpha)} \otimes_{A} c_{i(2, \beta)} \\
& =\sum_{i} n_{i(0)} \otimes_{B} n_{i(1)} \otimes_{B} b_{i} \otimes_{A} c_{i(1, \alpha)} \otimes_{A} c_{i(2, \beta)} \\
& =\sum_{i} n_{i} \otimes_{B} b_{i} \cdot \varphi\left(c_{i(1, \alpha)(1, e)}\right) \otimes_{A} c_{i(1, \alpha)(2, \alpha)} \otimes_{A} c_{i(2, \beta)} \\
& =\left(N \otimes_{B} \rho^{B \otimes_{A} C_{\alpha}} \otimes_{A} C_{\beta}\right)(x) .
\end{aligned}
$$

So $N \square_{D}\left(B \otimes_{A} C\right)=\left\{N \square_{D}\left(B \otimes_{A} C_{\alpha}\right)\right\}_{\alpha \in G}$ is an object of $\mathscr{M}^{G, C}$.

Take $f \in \operatorname{Hom}^{D}\left(M_{e} \otimes_{A} B, N\right)$, for all $\alpha \in G$ and $m \in M_{\alpha}$, since

$$
\begin{aligned}
\left(\rho^{N}\right. & \left.\otimes_{B}\left(B \otimes_{A} C_{\alpha}\right)\right) \circ \phi(f)_{\alpha}(m) \\
& =\left(\rho^{N} \otimes_{B}\left(B \otimes_{A} C_{\alpha}\right)\right)\left(f\left(m_{[0, e]} \otimes_{A} 1_{B}\right) \otimes_{B}\left(1_{B} \otimes_{A} m_{[1, \alpha]}\right)\right) \\
& =\left(f \otimes_{B} D \otimes_{B}\left(B \otimes_{A} C_{\alpha}\right)\right)\left(\rho^{M_{e} \otimes_{A} B}\left(m_{[0, e]} \otimes_{A} 1_{B}\right) \otimes_{B}\left(1_{B} \otimes_{A} m_{[1, \alpha]}\right)\right) \\
& =\left(f \otimes_{B} D \otimes_{B}\left(B \otimes_{A} C_{\alpha}\right)\right)\left(m_{[0, e][0, e]} \otimes_{A} 1_{B} \otimes_{B} \varphi\left(m_{[0, e][1, e]}\right) \otimes_{B}\left(1_{B} \otimes_{A} m_{[1, \alpha]}\right)\right) \\
& =\left(f \otimes_{B} D \otimes_{B}\left(B \otimes_{A} C_{\alpha}\right)\right)\left(m_{[0, e]} \otimes_{A} 1_{B} \otimes_{B} \varphi\left(m_{[1, \alpha](1, e)}\right) \otimes_{B}\left(1_{B} \otimes_{A} m_{[1, \alpha](2, \alpha)}\right)\right) \\
& =f\left(m_{[0, e]} \otimes_{A} 1_{B}\right) \otimes_{B} \varphi\left(m_{[1, \alpha](1, e)}\right) \otimes_{B}\left(1_{B} \otimes_{A} m_{[1, \alpha](2, \alpha)}\right) \\
& =\left(N \otimes_{B} \rho^{B \otimes_{A} C_{\alpha}}\right) \circ \phi(f)_{\alpha}(m) .
\end{aligned}
$$

Hence it follows $\phi(f)_{\alpha}(m) \in N \square_{D}\left(B \otimes_{A} C_{\alpha}\right)$. Conversely, let $f \in \operatorname{Hom}_{B}\left(M_{e} \otimes_{A}\right.$ $B, N)$. Assume that $\phi(f)_{\alpha}(m) \in N \square_{D}\left(B \otimes_{A} C_{\alpha}\right)$, by (3.1), it is sufficient to check that

$$
\phi\left(\rho^{N} \circ f\right)=\phi\left(\left(f \otimes_{B} D\right) \circ \rho^{M_{e} \otimes_{A} B}\right) .
$$

In fact, for all $\alpha \in G$ and $m \in M_{\alpha}$, since

$$
\begin{aligned}
\phi((f & \left.\left.\otimes_{B} D\right) \circ \rho^{M_{e} \otimes_{A} B}\right)_{\alpha}(m) \\
& =\left(f \otimes_{B} D\right) \circ \rho^{M_{e} \otimes_{A} B}\left(m_{[0, e]} \otimes_{A} 1_{B}\right) \otimes_{B}\left(1_{B} \otimes_{A} m_{[1, \alpha]}\right) \\
& =f\left(m_{[0, e][0, e]} \otimes_{A} 1_{B}\right) \otimes_{B} \varphi\left(m_{[0, e][1, e]}\right) \otimes_{B}\left(1_{B} \otimes_{A} m_{[1, \alpha]}\right) \\
& =f\left(m_{[0, e]} \otimes_{A} 1_{B}\right) \otimes_{B} \varphi\left(m_{[1, \alpha](1, e)}\right) \otimes_{B}\left(1_{B} \otimes_{A} m_{[1, \alpha](2, \alpha)}\right) \\
& =\left(N \otimes_{B} \rho^{B \otimes_{A} C_{\alpha}}\right) \circ \phi(f)_{\alpha}(m)
\end{aligned}
$$

and

$$
\begin{aligned}
\phi\left(\rho^{N} \circ f\right)_{\alpha}(m) & =\rho^{N}\left(f\left(m_{[0, e]} \otimes_{A} 1_{B}\right)\right) \otimes_{B}\left(1_{B} \otimes_{A} m_{[1, \alpha]}\right) \\
& =\left(\rho^{N} \otimes_{B}\left(B \otimes_{A} C_{\alpha}\right)\right) \circ \phi(f)_{\alpha}(m) .
\end{aligned}
$$

So we can get relation (3.2) from the assumption. 
Let us finally describe the unit $\eta$ of this adjunction in Prop. 3.5. Taking $M=\left\{M_{\alpha}\right\}_{\alpha \in G} \in \mathscr{M}^{G, C}$, the unit $\eta^{M}=\left\{\eta_{\alpha}^{M}\right\}_{\alpha \in G}$ for $(-)_{e} \otimes_{A} B \dashv-\square D\left(B \otimes_{A} C\right)$ at $M$ is given by

$$
\eta_{\alpha}^{M}(m)=\left(m_{[0, e]} \otimes_{A} 1_{B}\right) \square_{D}\left(1_{B} \otimes_{A} m_{[1, \alpha]}\right) .
$$

Now, we shall achieve the main goal in this section. Before presenting the main theorem, we first give the following remark which is necessary.

Remark 3.6. (1) Let $M=\left\{M_{\alpha}\right\}_{\alpha \in G} \in \mathscr{M}^{G, C}$. For each $\alpha \in G, \quad M^{\alpha}=$ $\left\{M_{\alpha \beta}\right\}_{\beta \in G}$ is a $G$-C-comodule via the structure map $\left\{\rho_{\beta, \gamma}^{M_{\alpha}}=\rho_{\alpha \beta, \gamma}^{M}\right\}_{\beta, \gamma \in G}$.

(2) Let $M=\left\{M_{\alpha}\right\}_{\alpha \in G} \in \mathscr{M}^{G, C}$ be flat as a right $A$-module. Then the map

$$
\left(M_{\alpha \beta} \otimes_{A} B\right) \otimes_{B}\left(B \otimes_{A} C_{\gamma}\right) \stackrel{\left(\rho_{\alpha, \beta}^{M} \otimes_{A} B\right) \otimes_{B}\left(B \otimes_{A} C_{\gamma}\right)}{\longrightarrow} M_{\alpha} \otimes_{A}\left(C_{\beta} \otimes_{A} B\right) \otimes_{B}\left(B \otimes_{A} C_{\gamma}\right)
$$

restricts to

$$
\begin{aligned}
\left(M_{\alpha \beta} \otimes_{A} B\right) \square_{D}\left(B \otimes_{A} C_{\gamma}\right) & \rightarrow M_{\alpha} \otimes_{A}\left(C_{\beta} \otimes_{A} B\right) \square_{D}\left(B \otimes_{A} C_{\gamma}\right) \\
& =\left(M_{\alpha} \otimes_{A} C_{\beta} \otimes_{A} B\right) \square_{D}\left(B \otimes_{A} C_{\gamma}\right)
\end{aligned}
$$

for all $\alpha, \beta, \gamma \in G$, where $M_{\alpha \beta} \otimes_{A} B, C_{\beta} \otimes_{A} B$ are considered as right $D$-comodules by applying the functor $(-)_{e} \otimes_{A} B$ to $M^{\alpha \beta}, C^{\beta}$, and $M_{\alpha} \otimes_{A} C_{\beta} \otimes_{A} B$ via $M_{\alpha} \otimes_{A} \rho^{C_{\beta} \otimes_{A} B}$.

THEOREM 3.7. Assume that $C$ is flat as a left A-module and every object in $\mathscr{M}^{G, C}$ is flat as right $A$-module. The functor $(-)_{e} \otimes_{A} B: \mathscr{M}^{G, C} \rightarrow \mathscr{M}^{D}$ is separable if and only if there is a family of homomorphisms of A-bimodule

$$
\theta=\left\{\theta^{(\alpha)}:\left(C_{\alpha^{-1}} \otimes_{A} B\right) \square_{D}\left(B \otimes_{A} C_{\alpha}\right) \rightarrow A\right\}_{\alpha \in G},
$$

such that

(1) $\theta^{(\alpha)} \circ \eta_{\alpha}^{C^{\alpha^{-1}}}=\varepsilon$

(2) $\theta$ satisfies the following commutative diagram:

$$
\begin{aligned}
\left(C_{\alpha^{-1}} \otimes_{A} B\right) & \square D\left(B \otimes_{A} C_{\alpha \beta} \stackrel{\left(\Delta_{\beta,(\alpha \beta)} \otimes^{-1} \otimes_{A} B\right) \otimes_{B}\left(B \otimes_{A} C_{\alpha \beta}\right)}{\longrightarrow} C_{\beta} \otimes_{A}\left(\left(C_{(\alpha \beta)^{-1}} \otimes_{A} B\right) \square_{D}\left(B \otimes_{A} C_{\alpha \beta \beta}\right)\right)\right. \\
\downarrow & \downarrow C_{\beta} \otimes_{A} \theta^{(\alpha \beta)} \\
\left(\left(C_{\alpha-1} \otimes_{A} B\right) \otimes_{B}\left(B \otimes_{A} \Delta_{\alpha, \beta}\right)\right. & C_{\beta}
\end{aligned}
$$

Proof. Assume that $(-)_{e} \otimes_{A} B$ is separable. By Rafael's Theorem (see [7]), there exists a natural transformation $\omega:\left((-)_{e} \otimes_{A} B\right) \square_{D}\left(B \otimes_{A} C\right) \rightarrow 1_{\mathscr{M}^{G, C}}$ such that $\omega \circ \eta=1$ (the identiy natural transformation.) Specially, considering $G-C$ comodule $C^{\alpha^{-1}}=\left\{C_{\alpha^{-1} \beta}\right\}_{\beta \in G}$ via $\Delta_{\alpha^{-1} \beta, \gamma}$ and applying $\omega$ to it, we have

$$
\omega^{C^{\alpha^{-1}}}=\left\{\omega_{\beta}^{C^{\alpha^{-1}}}:\left(C_{\alpha^{-1}} \otimes_{A} B\right) \square_{D}\left(B \otimes_{A} C_{\beta}\right) \rightarrow C_{\alpha^{-1} \beta}\right\}_{\beta \in G} .
$$


Then we construct a family of $k$-linear maps $\theta=\left\{\theta^{(\alpha)}\right\}_{\alpha \in G}$,

$$
\theta^{(\alpha)}=\varepsilon \circ \omega_{\alpha}^{C^{\alpha^{-1}}}:\left(C_{\alpha^{-1}} \otimes_{A} B\right) \square_{D}\left(B \otimes_{A} C_{\alpha}\right) \rightarrow A .
$$

From $\varepsilon$ and $\omega_{\alpha}^{C^{\alpha^{-1}}}$ being both right $A$-linear, it follows that the map $\theta^{(\alpha)}$ is a right $A$-module morphism. Next for all $a \in A$, we consider a family of $k$-linear maps $f^{\left(a, \alpha^{-1}\right)}=\left\{f_{\beta}^{\left(a, \alpha^{-1}\right)}\right\}_{\beta \in G}$,

$$
f_{\beta}^{\left(a, \alpha^{-1}\right)}: C_{\alpha^{-1} \beta} \rightarrow C_{\alpha^{-1} \beta}, \quad f_{\beta}^{\left(a, \alpha^{-1}\right)}(c)=a \cdot c .
$$

It is checked easily that $f^{a, \alpha}$ is a morphism of $\mathscr{M}^{G, C}$. By the naturality of $\omega$, we have the following commutative diagram

$$
\begin{aligned}
&\left(C_{\alpha^{-1}} \otimes_{A} B\right) \square_{D}\left(B \otimes_{A} C_{\beta}\right) \stackrel{\omega_{\beta}^{C^{\alpha}}}{\longrightarrow} C_{\alpha^{-1} \beta} \\
&\left(f_{e}^{\left(a, \alpha^{-1}\right)} \otimes_{A} B\right) \square_{D}\left(B \otimes_{A} C_{\beta}\right) \downarrow \downarrow f_{\beta}^{\left(a, \alpha^{-1}\right)} \\
&\left(C_{\alpha^{-1}} \otimes_{A} B\right) \square_{D}\left(B \otimes_{A} C_{\beta}\right) \stackrel{\omega_{\beta}^{C^{\alpha^{-1}}}}{\longrightarrow} C_{\alpha^{-1} \beta}
\end{aligned}
$$

for all $\alpha \in G$. It follows from the above commutative diagram that $\omega_{\beta}^{C^{\alpha^{-1}}}$ is left $A$-linear, thus $\theta^{(\alpha)}$ is left $A$-linear. Since

$$
\omega_{\alpha}^{C^{\alpha^{-1}}}\left(\left(c_{\left(1, \alpha^{-1}\right)} \otimes_{A} 1_{B}\right) \square_{D}\left(1_{B} \otimes_{A} c_{(2, \alpha)}\right)\right)=c
$$

for any $c \in C_{e}$, we have

$$
\begin{aligned}
\theta^{(\alpha)} \circ \eta_{\alpha}^{C^{\alpha^{-1}}}(c) & =\theta^{\alpha}\left(\left(c_{\left(1, \alpha^{-1}\right)} \otimes_{A} 1_{B}\right) \square_{D}\left(1_{B} \otimes_{A} c_{(2, \alpha)}\right)\right) \\
& =\varepsilon \circ \omega_{\alpha}^{C^{\alpha^{-1}}}\left(\left(c_{\left(1, \alpha^{-1}\right)} \otimes_{A} 1_{B}\right) \square_{D}\left(1_{B} \otimes_{A} c_{(2, \alpha)}\right)\right)=\varepsilon(c) .
\end{aligned}
$$

Now, for all $c \in C_{\beta}$, we consider the morphism

$$
l_{\gamma}^{(c, \alpha \beta)}: C_{(\alpha \beta)^{-1} \gamma} \rightarrow C_{\beta} \otimes_{A} C_{(\alpha \beta)^{-1} \gamma}, \quad c^{\prime} \mapsto c \otimes_{A} c^{\prime} .
$$

By the naturality of $\omega$, we have the following commutative diagram

$$
\begin{gathered}
\left(C_{(\alpha \beta)^{-1}} \otimes_{A} B\right) \square_{D}\left(B \otimes_{A} C_{\gamma}\right) \stackrel{\omega_{\gamma}^{C(\alpha \beta)^{-1}}}{\longrightarrow} C_{(\alpha \beta)^{-1} \gamma} \\
\left.\downarrow l_{e}^{(c, \alpha \beta)} \otimes_{A} B\right) \square_{D}\left(B \otimes_{A} C_{\beta}\right) \\
\left(C_{\beta} \otimes_{A} C_{(\alpha, \beta)^{-1}} \otimes_{A} B\right) \square_{D}\left(B \otimes_{A} C_{\gamma}\right) \stackrel{\omega_{\gamma}^{\Re(\beta, \alpha)}}{\longrightarrow} C_{\beta} \otimes_{A} C_{(\alpha, \beta)^{-1} \gamma}^{l_{\gamma}^{(c, \alpha \beta)}}
\end{gathered}
$$

where

$$
\mathfrak{R}^{(\beta, \alpha)}=\left\{\mathfrak{R}_{\gamma}^{(\beta, \alpha)}=C_{\beta} \otimes_{A} C_{(\alpha \beta)^{-1} \gamma}\right\}_{\gamma \in G} .
$$

It follows from the commutative diagram above that

$$
\omega_{\gamma}^{\Re^{(\beta, \alpha)}}\left(c \otimes_{A} x\right)=c \otimes_{A} \omega_{\gamma}^{C^{(\alpha \beta)^{-1}}}(x)
$$

for any $c \in C_{\beta}$ and $x \in\left(C_{(\alpha \beta)^{-1}} \otimes_{A} B\right) \square_{D}\left(B \otimes_{A} C_{\gamma}\right)$. Since $c$ is arbitrary, we have

$$
\omega_{\gamma}^{\Re^{(\beta, \alpha)}}=C_{\beta} \otimes_{A} \omega_{\gamma}^{C^{(\alpha \beta)^{-1}}} \text {. }
$$


The condition (2) in Theorem 3.7 follows from the following commutative diagram

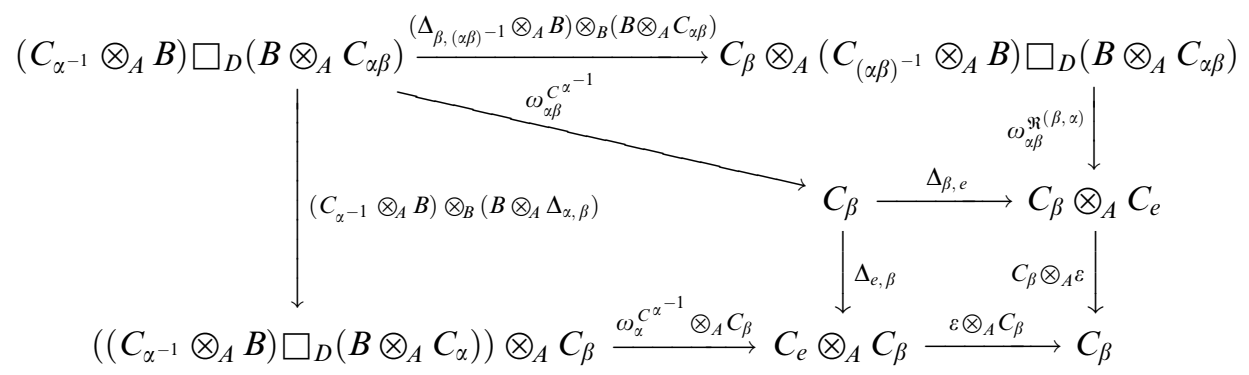

To prove the converse, we need to construct a natural transformation $\omega$ from the $A$-bimodule $\theta$. Given a right $G$-C-comodule $M=\left\{M_{\alpha}\right\}_{\alpha \in G}$, we define a family of $k$-linear maps $\omega^{M}=\left\{\omega_{\alpha}^{M}\right\}_{\alpha \in G}$, where $\omega_{\alpha}^{M}$ can be defined by the composition

$$
\begin{gathered}
\left(M_{e} \otimes_{A} B\right) \square_{D}\left(B \otimes_{A} C_{\alpha}\right) \stackrel{\left(\rho_{\alpha, \alpha^{-1}}^{M} \otimes_{A} B\right) \otimes_{B}\left(B \otimes_{A} C_{\alpha}\right)}{\longrightarrow} M_{\alpha} \otimes_{A}\left(\left(C_{\alpha^{-1}} \otimes_{A} B\right) \square_{D}\left(B \otimes_{A} C_{\alpha}\right)\right) \\
\stackrel{M_{\alpha} \otimes_{A} \theta^{(\alpha)}}{\longrightarrow} M_{\alpha}
\end{gathered}
$$

It follows from $\theta^{(\alpha)}$ being $A$-linear that each $\omega_{\alpha}^{M}$ is right $A$-linear. Using the following commutative diagrams

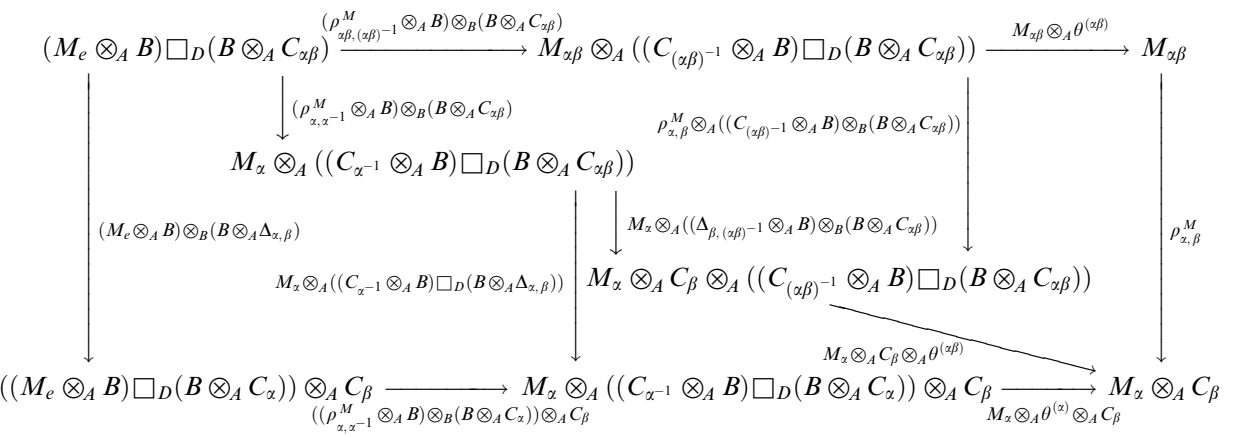

shows that $\omega^{M}$ is a morphism in $\mathscr{M}^{G, C}$, and

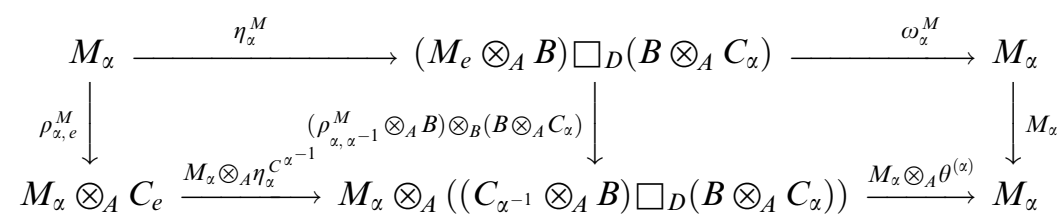

shows $\omega_{\alpha}^{M} \circ \eta_{\alpha}^{M}=i d_{M_{\alpha}}$. It is easily to check that $\omega$ is natural at $M$.

The proof is completed. 
By considering on $A$ the canonical $A$-coring structure, as a corollary of Theorem 3.7, we have the main result of [10].

COROLlary 3.8 ([10]). For a $G$-A-coring $\mathscr{C}$, the forgetful functor $F: \mathscr{M}^{G, C} \rightarrow \mathscr{M}_{A}$ is separable if and only if there exists a family of A-bimodules $\theta=\left\{\theta^{(\alpha)}: C_{\alpha^{-1}} \otimes_{A} C_{\alpha} \rightarrow A\right\}_{\alpha \in G}$ such that

$$
\begin{gathered}
\theta^{(\alpha)}\left(c_{\left(1, \alpha^{-1}\right)}^{\prime} \otimes_{A} c_{(2, \alpha)}^{\prime}\right)=\varepsilon\left(c^{\prime}\right), \\
c_{(1, \beta)} \cdot \theta^{(\alpha \beta)}\left(c_{\left(2, \beta^{-1} \alpha^{-1}\right)} \otimes_{A} d\right)=\theta^{(\alpha)}\left(c \otimes_{A} d_{(1, \alpha)}\right) \cdot d_{(2, \beta)}
\end{gathered}
$$

for all $c^{\prime} \in C_{e}, c \in C_{\alpha^{-1}}, d \in C_{\alpha \beta}$.

Acknowledgement. The authors would like to thank the referee for his/her valuable comments on this article.

\section{REFERENCES}

[1] T. BRZEZiŃSKI, The structure of corings, Induction functors, Maschke-type theorem, and Frobenius and Galois properties, Algebr. Represent Theory 5 (2002), 389-410.

[2] S. Caenepeel, K. Janssen and S. H. Wang, Group corings, Appl. Categ. Struct. 16 (2008), 65-96.

[ 3 ] S. Caenepeel and S. Raianu, Induction functors for the Doi-Koppinen unified Hopf modules, Abelian groups and modules, Kluwer Academic, Dordrecht, 1995, 73-94.

[4] X. L. FANG AND D. M. LU, Group corings and (invertible) weak group entwining structures, Comm. Algebra 36 (2008), 3820-3841.

[ 5 ] J. Gómez-Torrecillas, Separable functors in corings, IJMMS 30 (2002), 203-225.

[6] G. H. LiU And S. H. WAnG, Graded Morita theorem for group coring and graded MoritaTakeuchi theory, Taiwanese J. Math. 16 (2012), 1041-1056.

[7] M. D. RAfaEl, Separable functors revisited, Comm. Algebra 18 (1990), 1445-1459.

[8] M. E. SwEEdLER, The predual theorem to the jacobson-bourbaki theorem, Trans. Amer. Math. Soc. 213 (1975), 391-406.

[9] M. E. SweEdler, Hopf algebras, Benjamin, New York, 1969.

[10] D. G. WANG AND Q. G. Chen, Separable functor in group corings, to appear in Osaka J. Math.

Quan-guo Chen

School of Mathematics and Statistics

YILI NoRMAL UNIVERSITY

Yining, XinJIANG 835000

P.R. CHINA

E-mail: cqg211@163.com

Ding-guo Wang

SchoOl of Mathematical Sciences

QUFU Normal UNIVERSITY

Qufu, SHANDONG 273165

P.R. CHINA

E-mail: dingguo95@126.com 